\title{
Research of the Discipline Construction based on Colleges and Universities
}

\author{
Kexiang Tai \\ College of Humanities, Xi'an Technological University, \\ Xi'an 710021, China \\ e-mail: 285207738@qq.com \\ Yao Zhang \\ College of Humanities, Xi'an Technological University, \\ Xi'an 710021, China
}

\begin{abstract}
There are many misunderstanding in discipline construction between subject and discipline. There are many confusion with the concept of subject, degree and profession. This paper mainly research on the basic concept and the relationship of the three, the relationship between teaching and research, the basic task of colleges and universities. This paper point out the right way to cultivate a talent and lead to a right direction to the discipline construction.
\end{abstract}

Keywords- Discipline; Specialty; Concept Confusion

\section{INTRODUCTION}

There are three causes why we have to discuss this old topic again? Firstly, the many college teachers do not care about this topic and in their view, discipline construction is the leader's job; secondly, colleges and department level cadres although has been thinking about the matter, but it is very controversial, leading to the deviation in the implementation level; third, after the first batch of "double first class" list published, the solution of this problem is directly related to the long-term effectiveness of the unified cadres in colleges and the education idea of teachers and students of higher education or work.

What is the problem about the current university discipline construction? I believe that the answer of two: First, the concept confuses. Many people do not know what is "discipline" and what is "professional", and they are

\author{
Xiaoya Neng \\ College of Humanities, Xi'an Technological University, \\ Xi'an 710021, China \\ Zhichao Lian \\ College of Computer Science and Engineering, \\ Xi'an Technological University, \\ Xi'an 710021, China \\ e-mail: $965941167 @ q q . c o m$
}

confused about "discipline" and "degree"; of course, they also confused about the relationship of "discipline", "professional" and "degree"; Second, management imbalances. Many people directed the wrong direction to the work of colleges and universities rely on their fuzzy or wrong sense.

\section{RELATIONSHIP BETWEEN DISCIPLINES, MAJORS, DEGREES}

What is the discipline? According to the explanation of Hong Shimei and Fang Xing's, " there are two connotations: one is the classification of the academic, namely the scientific field or a scientific branch of science; The second is the abbreviation of teaching subject, namely the subject. [1] But it is not at the heart of discipline to this statement. however, there is another quote is almost close. "China's national standard GB / TI3735-92 defines the discipline as follows: 'discipline is relatively independent of the knowledge system'." [1] However, this definition is not specific and clear.

In my opinion, it is possible to say that discipline is a high-level knowledge system; in other words, subject is the theoretical induction and communication system of scientific research achievements. It is different from the industrialization of scientific research achievements. Advanced knowledge is corresponding to universal knowledge, the mission of higher education is to organize 
and disseminate advanced knowledge, secondary schools and the following are the dissemination of universal knowledge. In his book "higher education system", Clark devoted a special discussion to the problem of knowledge, and considered that advanced knowledge is the core of higher education system. [2] Therefore, it is not clear that the general understanding of discipline as a relatively independent knowledge system; at the same time, it is not comprehensive to equate the subject with scientific research, although the relationship between discipline and research is particularly close.

What is a majors? "Cihai" is defined as " the academic category that institutions of higher learning or secondary vocational schools divide according to the needs of the division of labor." Bieyuan Pan and others believe that " the major is an organizational form of the course." In Western higher education, "profession" refers to specialized fields with different ranges. The International Education Standard Classification called it a curriculum plan. [3]

I think this definition is also empty. Major is a necessary knowledge system for a career. It is not only includes the corresponding curriculum system, but also contains the theory of practice and inspection. Therefore, it is not very accurate to equate professional with teaching.

The relationship between discipline and major is that the discipline is the prerequisite for the major, which is the source of universal knowledge system. The content of the professional knowledge provided by the discipline, which should supported of many disciplines. Discipline knowledge is refined and corrected by the professional completion for that major is the application of disciplines, also is the promotion and popularization of the advanced knowledge system. To some content, disciplines for the professional menu, professional for the talent to transport energy. They are completely different from each other.

Degree is not the content of discipline construction and it is a problem on professional level. So, it is also a misunderstanding to equivalent the subject construction and construction of academic degrees. The basis of the discipline can not be ignored though the level of discipline is very important. Colleges will be mess up the connotation of the discipline construction and also easily lead the suspect of making achievement projects, if school leader blindly stressed the declaration and increase of master's and doctor's degree when the undergraduate level of the discipline has not yet qualified or the formation of characteristics. The flowchart of the subject construction is as shown in figure 1, It's work drawn by Hunan Institute of Science and Technology, it is not difficult to see that they really understand the subject construction as the declaration and construction of the graduate degree.

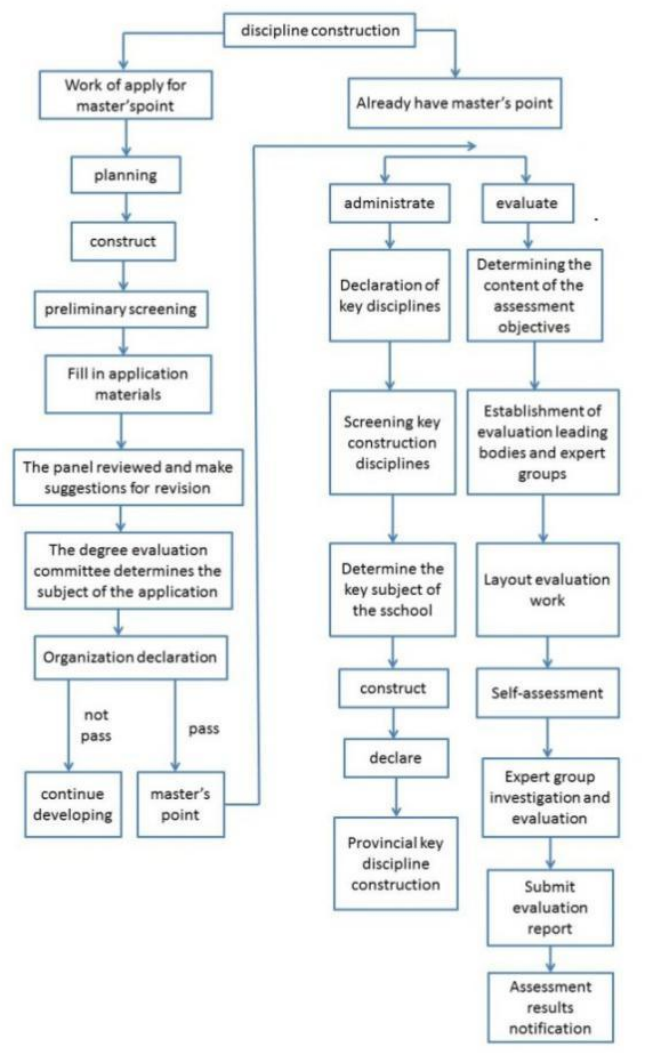

Figure 1 Flowchart of the Subject Construction

The construction of academic degrees is an important project in universities. It combines the discipline, professional, base construction and other factors. [4] To really need assisted in many departments of construction related degree, such as personnel (staff), logistics (property protection), base (platform), object (enrollment and employment) and so on, however, the discipline construction is always the center of scientific research and teaching, 
construction of degree can't be equivalent to the discipline construction.

Just like the college entrance examination of the baton for middle school is difficult to reverse. Some people may say that the department of education's discipline evaluation mechanism and the indicators should be consider. This worry is naturally right, the system is difficult to suddenly change things as people criticize the same college entrance examination. However discipline construction need spend long time to complete and require everyone honestly one step a footprint and can't get rich quick. [5] Therefore, in the dominant ideology, we must respect the basic law of the natural growth of the discipline.

If we emphasize discipline only, its focus on scientific research; emphasis on specialty and centre on teaching. Concept of "discipline construction", actually contains the research and teaching of the connection. Therefore, the ultimate goal of discipline construction is still in the cultivation of talents. This is a virtuous cycle that colleges will be able to attract more students apply who are interested in studying the advanced knowledge and professional will be expand or it can create talent for social if the professional were so well .

\section{THE RELATIONSHIP BETWEEN TEACHING AND RESEARCH}

In the first place, the university is institution of education. Its scientific research function directly serves the teaching or talents cultivation. Therefore, the scientific research work of a university is different from the research work of the social institution. The main purpose of the latter is to the industrialization of scientific research, and the focus of university scientific research work is to the theorization of scientific research achievements, Of course, do not rule out the industrialization of certain results

The process of theorization of scientific research achievements firstly of all is that gradually turn some of the original scientific research achievements ( papers, writings, etc.) into special academic reports, and then be opened in a range of elective courses, further evolved into compulsory and continuous countless related courses, finally formed a mature knowledge system in a particular field or direction.

One of the concrete manifestations of theorization of scientific research achievements is compilation of high level teaching materials, especially the compilation of new subject textbooks. To some extent, it is a higher scientific research, because it integrate the latest and most mature research results in an area, which is the direct embodiment of the application of scientific research achievements in Teaching. In other words, the task of discipline construction is to gradually improve and promote the development of existing disciplines from the creation and teaching of numerous related new courses. And on this basis, through the integration of traditional disciplines giving birth to a new subject, it will produce its byproducts: scientific research (including the results of the theory and practice). In short ,the process of discipline construction is "scientific research -Teaching -- scientific research".

For that reason, the university can always become the birthplace of knowledge innovation. Moreover, in another sense, the promotion and popularization of scientific research achievements is a clear proof of the industrialization of scientific research achievements in universities.

\section{THE BASIC TASK OF THE UNIVERSITY IS THE CULTIVATION OF TALENT}

The training and supply of talents is the main contribution of universities to society. Nowadays, The state is looking forward to more and more functions of the university, such as social service, cultural heritage, scientific research and so on, which is also the basic task of the University. But in comparison, talent training is always the most important mission of the university. The basic purpose of discipline construction is to serve talents cultivation.

The basic concepts are clarified so that consensus, unified thinking and cohesive forces can be formed. If the administrative work of the university is well done, the orientation of the university will not deviate, and the focus of discipline construction will be highlighted.

The level of discipline construction is a manifestation of the core competence of University. To do a good job of 
discipline construction must first respect rules of discipline structure, based on mastering discipline internal elements, focus on cross-integration between disciplines, meet the needs of social development and change, and constantly explore new knowledge systems and create new disciplines, at the same time, formulate and perfect the corresponding discipline organization and its guarantee system, especially form the discipline culture of each university in order to highlight the discipline characteristic.

Each university has its value of independent existence, and even has a wide range of social appeal, and relating to a fame of masters. But the fame of the great masters is not out of the question, precisely because some of their research, even a paper and a book, caused numerous courses, and even led to the birth of a new discipline. Therefore, the work of discipline construction at the operational level is to advocate and encourage the majority of professors to actively transform their original research results into courses. Attention please, what I emphasize here is originality rather than sorting and retelling.

A university may have created several masters due to several influential courses, and have formed their own system or style and school in the discipline construction because of some mature and advanced courses. The University describes its subject culture in fashionable words, so when someone mentions a certain subject, he can immediately think of XX university.

Don't want many subjects can achieve this goal, as long as there are one or two famous discipline or direction, and whether it is engineering, liberal arts or science. It is the real name card of the school, rather than relying on boasting, self styled, and self etching bubbles. Naturally, to achieve this goal, a university may require several generations of struggle unremittingly, but it is necessary and worthwhile.

Above, I just want to show my superficial understanding. We can get a lot of argument by referencing to the academic research results, here does not enumerate. The main purpose of this article is to hope that colleagues to rethink and explore.

\section{REFERENCE}

[1] Hong Shimei, Fang Xing, theoretical clarification of several related concepts in subject construction, higher education development and evaluation, March 2006.

[2] (USA) Clark. Higher education system[M]. Wang Chengxu translation. Hangzhou University Press, 1994: 12

[3] Chen Hongjie, comments on advanced knowledge and higher education, Peking University Education Review, No. 4, 2006.

[4] http://xk.hnist.cn/show.asp, id=144\#, website of Graduate School of Hunan Institute of Science and Technology, subject construction column: the concept of discipline construction ; the process of discipline construction.

[5] Su Junping, chief editor of Jiang Bei, subject and discipline construction, Second Military Medical University press, October 2014 